University of Nebraska - Lincoln

DigitalCommons@University of Nebraska - Lincoln

Faculty Publications, Department of Psychology

Psychology, Department of

2006

\title{
Effects of temporal clumping and payoff accumulation on impulsiveness and cooperation
}

\author{
David W. Stephens \\ University of Minnesota - Twin Cities, dws@forager.cbs.umn.edu \\ Colleen M. McLinn \\ University of Minnesota - Twin Cities \\ Jeffrey R. Stevens \\ University of Nebraska-Lincoln, jstevens5@unl.edu
}

Follow this and additional works at: https://digitalcommons.unl.edu/psychfacpub

Part of the Psychiatry and Psychology Commons

Stephens, David W.; McLinn, Colleen M.; and Stevens, Jeffrey R., "Effects of temporal clumping and payoff accumulation on impulsiveness and cooperation" (2006). Faculty Publications, Department of Psychology. 538.

https://digitalcommons.unl.edu/psychfacpub/538

This Article is brought to you for free and open access by the Psychology, Department of at DigitalCommons@University of Nebraska - Lincoln. It has been accepted for inclusion in Faculty Publications, Department of Psychology by an authorized administrator of DigitalCommons@University of Nebraska - Lincoln. 


\title{
Effects of temporal clumping and payoff accumulation on impulsiveness and cooperation
}

\author{
David W. Stephens, Colleen M. McLinn, and Jeffrey R. Stevens \\ Ecology, Evolution and Behavior, University of Minnesota, St. Paul, MN 551108, USA \\ Corresponding author - D. W. Stephens; tel 612 625-5722, fax 612 624-6777, email dws@forager.cbs.umn.edu
}

\begin{abstract}
Animals show impulsiveness when they prefer a smaller more immediate option, even though a larger more delayed option produces a higher intake rate. This impulsive behavior has implications for several behavioral problems including social cooperation. This paper presents two experiments using captive blue jays (Cyanocitta cristata) that consider the effects of payoff accumulation and temporal clumping on impulsiveness and cooperation. Payoff accumulation refers to a situation where the benefits gained from each choice trial accumulate from one trial to the next, and only become available to the animal after it has completed a fixed number of trials. We hypothesized that this would reduce impulsiveness because it removes the advantage of quickly realizing food gains. Clumping refers to situation in which the animal experiences several choice trials in quick succession followed by a long pause before the next clump. We hypothesized that if payoffs accumulated over a clump of trials this would enhance the effect of accumulation. We tested the effects of accumulation and clumping on impulsiveness in a self-control situation. We found a significant interaction between clumping and accumulation. Payoff accumulation reduced impulsiveness, but only when trials were clumped. Post hoc analyses suggest that clumping alone increases impulsiveness. A second experiment applied these results to cooperation. This experiment reveals an interaction between payoff accumulation and trial's position within the clump. Jays were more likely to cooperate on the first trial of a clump, but the likelihood of cooperation dropped after the first trial. However, this drop was larger when payoffs did not accumulate. This observation suggests that the difference between accumulated and unaccumulated treatments that we reported previously (Science 198: 2216-2218) may be largely due to differences in how animals behave in the first trial of a clump.
\end{abstract}

Keywords: impulsivity, cooperation, choice, temporal clumping, payoff accumulation, prisoner's dilemma

\section{Introduction}

Animals show impulsiveness when they prefer an immediate food reinforcer even though a more delayed alternative yields a higher rate of food intake. This preference for immediacy could have important implications for behavioral models of cooperation, like the famous Iterated Prisoner's Dilemma, which focus on situations in which defecting (that is, not cooperating) leads to an immediate gain. Indeed some workers have argued that exhibiting self-control rather than impulsiveness is almost identical to cooperating rather than defecting (Platt, 1973; Rachlin, 2000, 2002). To cooperate in situations like the Prisoner's Dilemma, animals must forego the immediate temptation to defect in anticipation of a larger stream of gains in the long run. Since, by definition, impul- sive animals prefer immediate rewards, strong impulsiveness may present a significant barrier to cooperation.

Beyond this theoretical link, there is an intriguing empirical parallel between impulsiveness and cooperation. In both topics, human and non-human results disagree. Experiments suggest that non-human animals are much more impulsive than humans (Mazur, 1987; Rachlin et al., 1991; Richards et al., 1997), and much less likely to cooperate in experimental games (Clements and Stephens, 1995; Sally, 1995). This correlation agrees with our predictions: subjects that are more impulsive should be less cooperative (see Harris and Madden, 2002 for evidence of this correlation in human subjects). However, we must view this correlation with some caution because human and non-human studies use quite different procedures (Jackson and Hackenberg, 1996). 
Our goal was to investigate the idea that strong impulsiveness in animals prevents cooperation in experimental Prisoner's Dilemmas. To test this, one would like some manipulation or treatment that switches impulsiveness off. With such a treatment, we should be able to show that animals cooperate in a Prisoner's Dilemma when we experimentally "switch off" their impulsiveness, but defect otherwise. The difficulty here is that we know relatively little about the causes of impulsiveness, although the literature gives several examples of impulsiveness varying across contexts in both humans and non-humans (Mazur, 1994; Mischel et al., 1989; Stephens and Anderson, 2001; Stephens and McLinn, 2003; Wilson and Daly, 2004). A manipulation that should, in theory, have a large effect on impulsiveness is payoff accumulation (Stephens, 2000).

\subsection{Accumulation}

In a typical choice experiment, an animal makes a choice and receives the benefits of its decision, and this pattern of choice and reward continues over many trials. Imagine instead that the benefits of each choice accumulate over a sequence of trials, becoming available for collection only after the subject completes four trials (for example). If impulsiveness occurs because more immediate reinforcers are more valuable, then payoff accumulation should reduce or eliminate impulsiveness because it eliminates the advantage of immediacy. There should be no advantage to choosing the more immediate option because the subject must wait for all its accumulated gains. We present two experimental studies that explore the potential of accumulation to influence impulsiveness. The first experiment directly asks whether accumulation reduces impulsiveness, and the second asks whether accumulation increases cooperativeness.

\subsection{Clumping}

When we say that the bushes in a garden are clumped, we mean that several bushes are clustered together and clearly separated from other clumps. By analogy, this paper refers to events that are clumped in time. When events are clumped in time, groups of events occur in quick succession, but relatively long intervals separate one group from another. We reasoned that if some process created temporal clumps of trials, this would make accumulation more evident to the subjects, and possibly enhance accumulation's effect on impulsivity. The rationale of this claim is that when trials are clumped and accumulated the subject experiences a quick sequence of trials separated by a long interval, collecting reinforcement after every clump of trials. The long gap between clumps should make it easier to recognize clumps of trials, and we reason that it may also make it easier to recognize that payoff accumulation combines the benefits derived from these clumps. In contrast, if accumulated trials were equally spaced in time, this may blur the distinction between one set of accumulated trials and the next, and so possibly reduce the effect of accumulation. Our first experiment factorially combined accumulated and unaccumulated treatments with clumped and unclumped treatments using a con- ventional impulsiveness test. This experiment offered subjects a choice between a small immediate and a large delayed option using an experimental situation that psychologists call the self-control paradigm.

Experiment 2 applied the results of experiment 1 to cooperation by testing blue jays in an experimental Prisoner's Dilemma with and without accumulation. We reported the main effects of accumulation on cooperation elsewhere (Stephens et al., 2002). Here, we focus on patterns related to clumping that we have not reported previously.

\section{General methods}

\subsection{Double-V apparatus}

The apparatus used in both experiments had two side-byside compartments, each shaped like a "V" (Figure 1A). Each compartment was equipped with three perches, one in the rear and two in the front. Each perch was positioned immediately below a stimulus light (Med Associates ENV-124AM) that could display any of several colors. A microswitch connected to each perch allowed the experimental program to detect the presence or absence of a bird. Generally speaking, the subject waited on the rear perch when the associated rear light illuminated, and hopped forward to make a choice when the front lights illuminated.

The front panel of each compartment was also equipped with a food cup, a 20-mg pellet feeder (Med Associates ENV203-20), and a custom-made device that we call the accumulator (Figure 1B). The accumulator was a small box made of transparent plastic. The pellet dispenser delivered pellets into the accumulator so that the subject could see the food pellets it had earned but not consume them. At experimentally determined times, a flap on the bottom of the accumulator opened (by energizing a solenoid), delivering food into the food cup. The accumulator allowed us to create treatments in which food accumulated, seen but unavailable, over several trials, or unaccumulated treatments in which the apparatus dispensed food immediately at the end of a trial.

\subsection{Training}

Before testing subjects in either experiment, we trained them to perform a task we call "light following." In light following, the subjects waited on the rear perch for a fixed time, then hopped forward to the front perch associated with an illuminated stimulus light. Each training trial presented only one of the two front perches, and the order of left and right presentations was selected randomly. During this training, we trained the subject to visit both the left and right perches. This training used conventional shaping techniques, and typically took 3-4 weeks to complete.

Subjects spent $23 \mathrm{~h}$ in the apparatus, obtaining all of their food there. We removed the subjects from the apparatus for $1 \mathrm{~h}$ each day (11:00-12:00). During this break, we weighed the subjects, and cleaned and tested the apparatus. Experimental contingencies were in force $8 \mathrm{~h}$ per day: starting at 


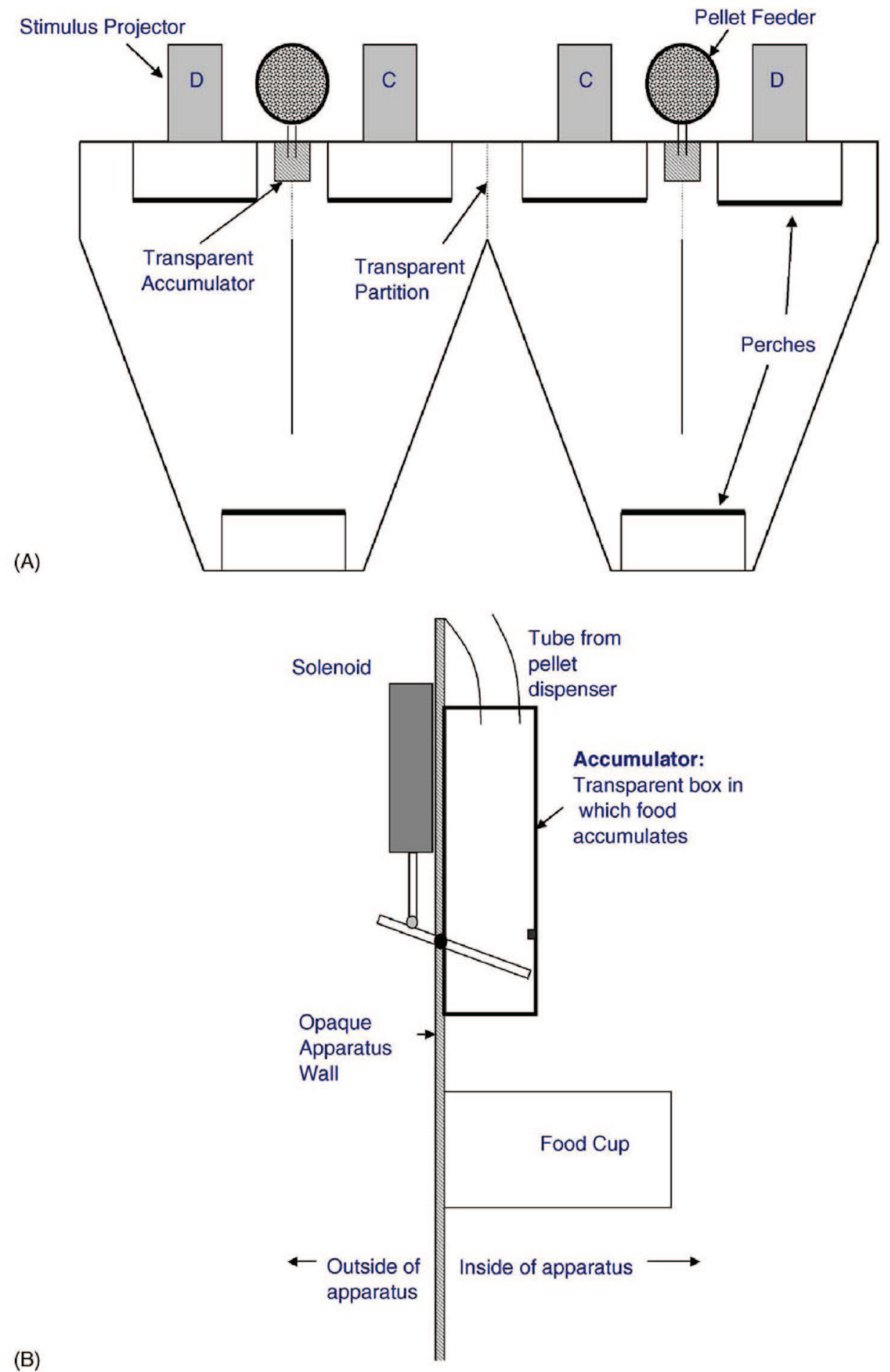

Figure 1. (A) Plan of apparatus. The apparatus consisted of side-by-side V-shaped compartments. Each compartment had three perches and three stimulus projectors. (B) The accumulator. To create accumulated and unaccumulated treatments, we fixed a transparent box to the front of each compartment as shown here. A standard pellet dispenser delivered food into this box. In accumulated treatments, pellets accumulated in the box over several trials until we energized a solenoid that opened a flap at the bottom of the accumulator.

(B)

07:00, stopping for the break at 11:00, starting again at 12:00 and ending for the day 16:00. All stimulus lights were dark during the periods when contingencies were not in force. We provided supplementary food (at 16:00 daily) for any bird that obtained less than $7 \mathrm{~g}$ during the day. A white noise generator provided masking noise whenever the experimental contingencies were in force.

\subsection{Statistical methods}

Both experiments followed a within-subjects or repeated measures design. To assess effects of time, we divided the free trials into three time blocks - i.e., first third of the data, second third, third third - and analyzed the proportional choice for each third. When a plot or analysis called for a 
single measure of performance, we used the data from the last third of free trials for a given treatment. We used conventional repeated measures ANOVA using arcsine transformed choice proportions to analyze the data.

\section{Experiment 1}

The goal of this experiment was to determine whether and under what conditions accumulation and the temporal arrangement of trials influence blue jay preferences for immediacy. The experiment followed the self-control procedure typically used in discounting studies (e.g., Rachlin and Green, 1972). In self-control studies, subjects must choose between a small-immediate benefit and a large-delayed benefit.

\subsection{Experiment 1-Methods}

\subsubsection{Overview}

The sequence of events within a trial was as follows: (1) the subject waited for a fixed time (the inter-trial interval or ITI); (2) the apparatus offered a choice between small-immediate and large-delayed options by illuminating lights of different colors at the front of the apparatus; (3) the subject chose one of the options by hopping forward to the associated perch. Once the subject made its choice, the unchosen light was switched off and the experimentally programmed delay to food began; (4) when the programmed delay expired, the apparatus dispensed food and the process began again at step 1. In this experiment, the small-immediate option offers one $20 \mathrm{mg}$ food pellet and the large-delayed option offers three $20 \mathrm{mg}$ food pellets. The delays varied according to experimental treatments as described below.

\subsubsection{Subjects}

The subjects in experiment 1 were six adult blue jays of unknown sex and mixed experimental histories: band numbers b70, b85, b108, b223, b229 and b239.

\subsubsection{Stimulus colors}

We selected the light colors associated with small-immediate and large-delayed randomly for each subject from the set: yellow/ blue, blue/yellow, green/red and red/green. We maintained this association for each subject throughout the experiment.

\subsubsection{Treatments}

We organized trials into blocks of 32 , the first eight trials within each block were forced or "no choice" trials in which the subjects was offered only one option (i.e., either the small-immediate option or the large-delayed option, but not both). The remaining 24 trials were free choice trials, in which subjects could choose between the two options.

\subsubsection{Baseline treatments}

In order to minimize order effects, each individual experienced a baseline treatment before being tested in each clumping/accumulation combination. We designed the baseline treatment to be intermediate between the actual experimental treatments. The delay-to-large was $60 \mathrm{~s}$ and the delay-to-small was $25 \mathrm{~s}$. Within this treatment, subjects experienced a random mix of all four of the accumulation/ clumping combinations. At the beginning of each set of four trials, the baseline program randomly determined whether the next four trials would be: (a) clumped or not and (b) accumulated or not. In clumped trials, the subject waited $285 \mathrm{~s}$ before being presented with a succession of four trials, one 5 $\mathrm{s}$ after the other. In non-clumped trials, the subject waited 75 $\mathrm{s}$ between each of the four trials. Subjects experienced 4 days of these baseline trials before starting each new clumping/ accumulation combination.

\subsubsection{Clumping treatments}

We arranged trials into groups of four (within the blocks of 32 discussed above). In temporally "clumped" treatments, the subject experienced a $345 \mathrm{~s}$ inter-trial interval between clumps and then experienced a quick succession of four trials, with a $5 \mathrm{~s}$ inter-trial intervals, totaling four trials every $360 \mathrm{~s}$. In unclumped trials, the subject waited $90 \mathrm{~s}$ between each trial, again experiencing four trials every $360 \mathrm{~s}$. Expressing this another way, the inter-trial interval varied in clumped treatments (sometimes $5 \mathrm{~s}$ and sometimes $345 \mathrm{~s}$ ), but was fixed in unclumped treatments (always $360 \mathrm{~s}$ ).

\subsubsection{Accumulation treatments}

In accumulated treatments, food remained in the accumulator - visible to the subject, but unavailable - for four trials, then the accumulator opened delivering food immediately after the fourth trial was completed. In unaccumulated treatments, the accumulator opened immediately after the pellet dispenser delivered the last pellet of food.

\subsubsection{Delay treatments}

To assess whether our manipulations influenced the blue jays' sensitivity to delay we needed to vary the delays associated with large (three $20 \mathrm{mg}$ food pellets) and small (one $20 \mathrm{mg}$ food pellet) options. Therefore, we tested each clumping/accumulation combination at six different delay-tolarge/delay-to-small combinations. We tested two delays-tolarge (45 and $75 \mathrm{~s}$ ) and three delays-to-small (5, 15 and 30 s). Each subject, therefore, experienced 24 distinct treatments (two levels of accumulation, two levels of clumping, two levels of delay-to-large and three levels of delay-to-small). Each subject experienced the four accumulation/ clumping treatments in a randomly determined order. In addition, we randomized the order of the six delay treatments within each accumulation/clumping treatment, so that a given individual experienced all six delay treatments within a given level of accumulation and clumping before moving on to the next, randomly determined, accumulation/clumping combination. Each treatment ran for 3 days.

\subsection{Experiment 1-Results}

Figure 2 shows an overview of the data. The figure shows proportional choice of the large-delayed alternative called $P$ (Large) in the figure, for each bird and each condition of the experiment. It is somewhat difficult to see the patterns in the data in this graph, but one does see considerable individual variability in the data. For example, we see that all in- 


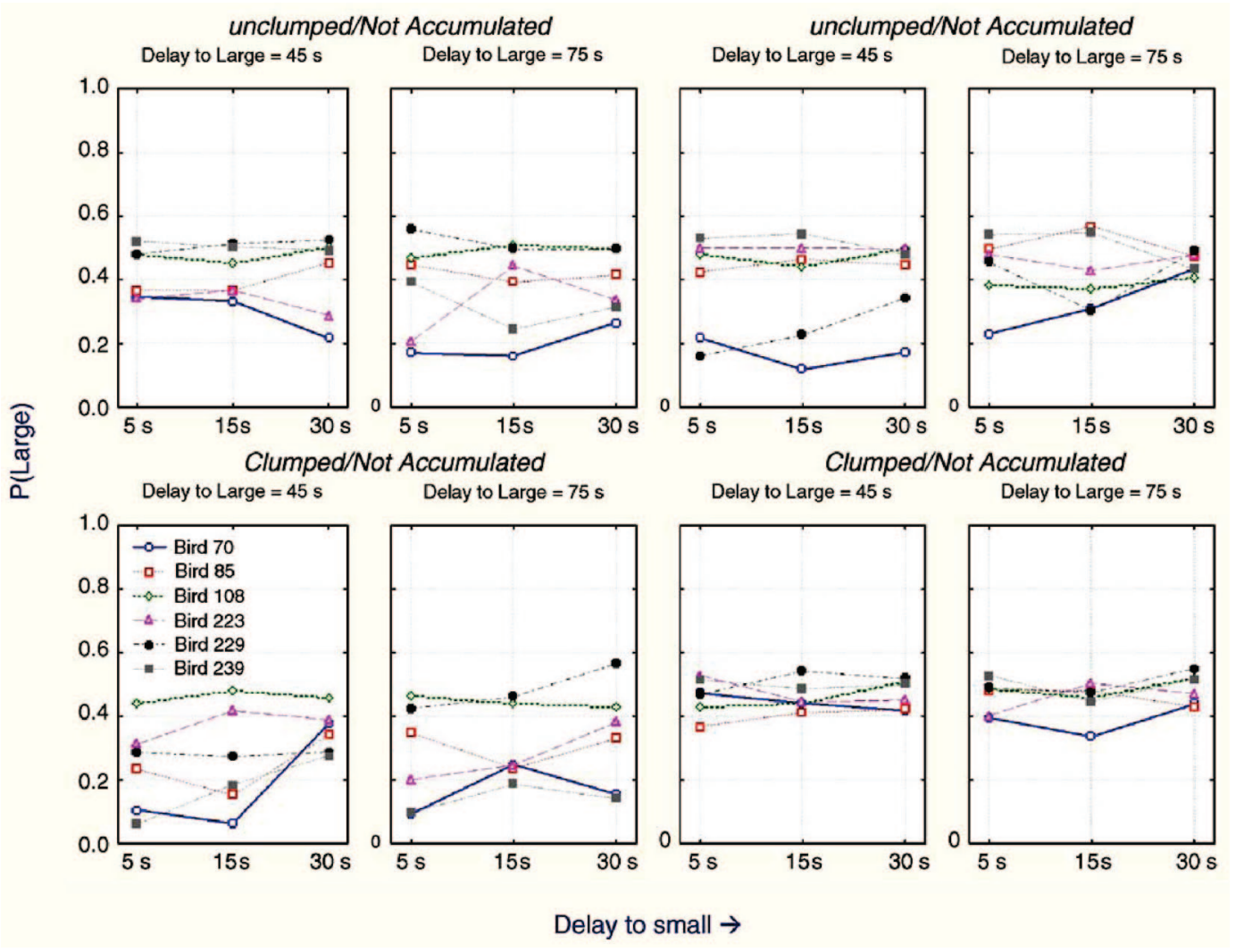

Figure 2. Overview of experiment 1. Each panel shows the relative frequency with which the subject choose the large-delayed alternative [denoted by $P$ (Large)] for each of six subjects as a function of the delay to small. These plots show $P$ (Large) calculated from the final third of free trials for each treatment. The eight separate panels each show a different combination of delay to large (45 or 75 s), clumping (clumped or not clumped) and accumulation (accumulated or not accumulated).

dividuals are nearly indifferent in the "clumped and accumulated" treatment, while there is much more variability in other treatments. We also see that indifference $(P($ Large $)=$ 0.5 ) represents an upper bound in the data; when a subject shows a strong preference, it is a preference for the small-immediate option.

Repeated measures analysis of variance on accumulation, clumping, delay-to-small and delay-to-large treatments shows two significant effects: a significant accumulationclumping interaction $\left(F_{1,5}=9.9, p=0.025\right)$ and a significant effect of delay-to-small $\left(F_{2,10}=6.87, p=0.025\right)$.

Figure 3 shows the pattern of the clumping-accumulation interaction. The "clumped and not accumulated" treatment shows a significant shift toward choice of the small-immediate option (i.e. small $P($ Large)); while accumulation clearly makes no difference when trials are unclumped. The "accumulated and clumped" appears to be slightly elevated but further analyses suggest that this is not significant. Post hoc analysis show significant differences between the "clumped and not accumulated" treatment and all others, but no differences between the three remaining treatments. In addition, we observed a trend for an accumulation main effect $\left(F_{1,5}=5.4, p=0.067\right)$, but we focus on the significant interaction demonstrating how clumping modulates accumulation Figure 4 shows the significant main effect of delay-to-small; large delays-to-small increase proportional choice of the large-delayed option $(P($ Large $))$. It is somewhat surprising that we did not find an effect of the logically similar treatment variable delay-to-large, but the range of delay-to-large values tested here might have been too small.
When trials were clumped trials in time, subjects may have made different choices on the first and fourth trials within a clump. To account for this effect, we recalculated our analysis of variance including "clump position" as a factor. This analysis shows no effect of a trial's position within a clump. A graph of $P$ (Large) versus clump position shows no hint of a trend. We report this negative result because experiment 2 shows a striking effect of clump position.

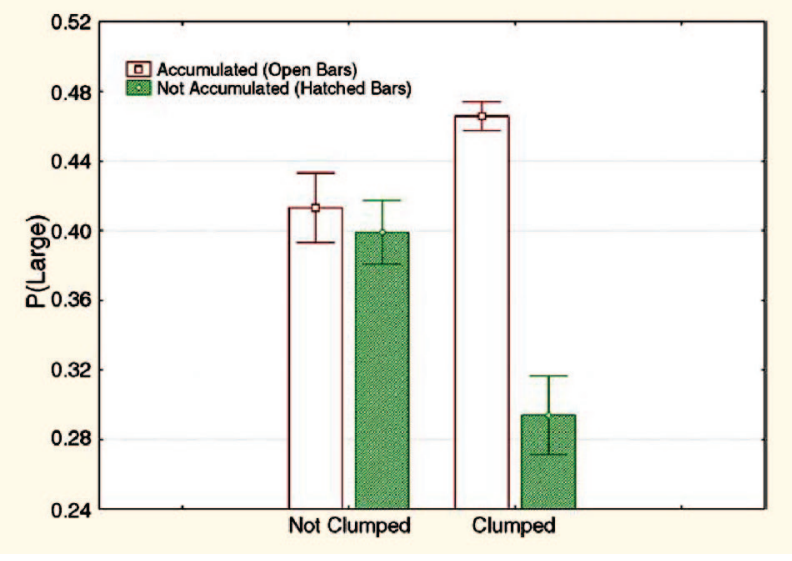

Figure 3. An interaction plot showing the combined effects of clumping and accumulation. The whiskers show standard errors. Repeated measures analysis of variance showed that this interaction was significant $\left(F_{1,5}=9.9, p=0.025\right)$. As in Figure 2 , this plot summarizes data from the final third of free trails for each treatment. 


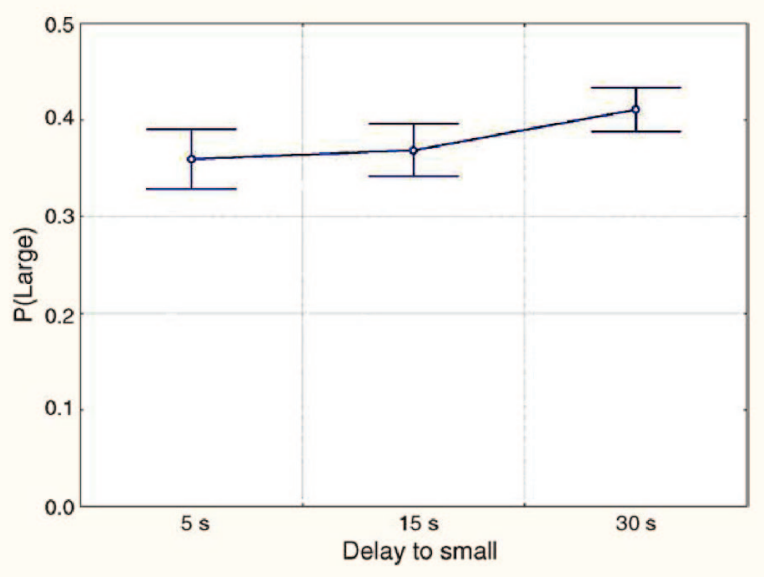

Figure 4. The significant effect of delay-to-small in experiment 1 . Preference for the large-delayed option $[P($ Large $)]$ increased when we increased the delay associated with the small option $\left(F_{2,10}=6.87, p=\right.$ 0.025). As in previous figures, this plot summarizes data from the final third of free trails for each treatment.

The interaction between accumulation and temporal clumping is very intriguing (Figure 3). We introduced accumulation treatments because we hypothesized that they would reduce impulsiveness, and indeed, we found that blue jays were less likely to choose the small-immediate option in accumulated treatments, but only when we clumped trials in time. Our results suggest that clumping increases impulsiveness and accumulation reverses this effect. The reader should recall that we conduct our studies at a longer time scale than typical non-human studies of impulsiveness and self-control. For example, in our unclumped treatment subjects experienced a $90 \mathrm{~s}$ inter-trial interval for each trial over an $8 \mathrm{~h}$ period.

\subsection{Experiment 1-Discussion}

This unexpected role of temporal clumping is significant in several ways. First, temporal clumping is a new factor in studies of impulsivity. Variables such as the overall delay to reward are analogous to clumping in that they influence the temporal arrangement of benefits (e.g., Rachlin and Green, 1972). Although earlier studies of impulsiveness have implicated similar variables, they focus on the temporal properties of the alternatives offered (see, for example, Bateson and Kacelnik, 1996; Green, 2000; Mazur and Logue, 1978; Mazur et al., 1985). To create clumps, we varied the temporal arrangement of the choices, while holding the delays and amounts associated with each alternative fixed. Indeed, a long-standing claim of the self-control literature is that the duration of the inter-trial interval (the time between choices) does not affect impulsiveness (Bateson and Kacelnik, 1996; Gallistel and Gibbon, 2000). We achieve clumping, obviously enough, by varying the inter-trial intervals. In unclumped treatments, inter-trial intervals are always the same, while inter-trial intervals are sometimes short and sometimes long in clumped treatments. So, our results suggest that variability in the inter-trial interval may affect impulsiveness, even if the magnitude of the inter-trial interval does not. Although the generality of our clumping result needs further investigation, we think it worth asking whether the short sessions of typical self-control studies may represent "clumps" that enhance impulsiveness. If so, this might partially explain why non-human subjects seem so absurdly impulsive.

Our clumped treatments raise questions about how one should calculate the intake rates; since the rate per trial varies from one trial to the next. The general discussion addresses this question, and reviews relevant literature from behavioral ecology.

Many experimental studies have investigated animal impulsiveness. These studies have emphasized the properties of the alternatives that the experiment offers the animal. For example, an important sequence of studies shows that increasing both delays (the delay-to-small and delay-to-large) by the same amount shifts preference toward the larger, more delayed alternative (Green et al., 1981; Rachlin and Green, 1972). This preference reversal effect is one of the fundamental facts of animal impulsiveness. Similarly, several authors have compared randomly varying delays to fixed delays with the same mean duration (Mazur, 1989; Rachlin et al., 2000). In these experiments, animals show a preference for variable delays. Students of impulsiveness see this as further evidence that a decreasing concave-up function governs the subjective value of delayed food. The clumping variable that we explore here is unusual because it is not a property of the alternatives themselves. Instead clumping refers to the arrangement of alternatives in time. There has been comparatively little work on how such aspects of the "background" influence subjective choice. Indeed, the literature gives the overall impression that any "background" effects on choice and impulsiveness are virtually non-existent. One exception to this is Mazur's (1994) demonstration that dispensing food between choices (i.e., within the inter-trial interval) shifts choice toward the small-immediate alternative. Our result shows a similar shift, but it is not clear that our clumping treatments represent background enrichment because the average long-term rate of intake is the same in clumped and unclumped treatments (see general discussion for review of rate calculations). Our results may be similar to Mazur's background enrichment effect if we assume that variability in the temporal arrangement of the background (clumping) increases the value of the background in the same way that variability in delays to food delivery makes alternatives in the foreground more attractive. We remark, however, that our clumped treatments represent deterministic rather than stochastic variation in the background of a particular pair of alternatives.

Payoff accumulation superficially resembles reward bundling (Ainslie and Monterosso, 2003; Brunner and Gibbon, 1995; Mazur, 1986; Mitchell and Rosenthal, 2003). In bundling studies, a single choice leads to multiple delayed rewards, for example, a pecking a green key might lead to two pellets after $10 \mathrm{~s}$ followed by an additional two pellets after an additional $10 \mathrm{~s}$. In accumulation, in contrast, subjects make a sequence of decisions that combine to influence the magnitude and timing of a single delayed reward. Qualitatively, bundling and accumulation appear to have similar effects in that they can both shift choice away from smaller more immediate options (Ainslie and Monterosso, 2003). Quantitatively, we have much more information about bun- 
dling, where evidence suggests that the discounted value of a sequence of rewards is simply of the sum of their separately discounted values (e.g., Brunner and Gibbon, 1995; Mazur, 1986). It is less clear how one would apply discounting models to the many actions, one outcome case posed by accumulation.

\section{Experiment 2}

\subsection{Overview}

We designed this experiment to study the interaction between discounting and opponent strategy in an Iterated Prisoner's Dilemma. Experiment 1 showed that accumulation increased proportional choice of a large-delayed option when trials are clumped. We, therefore, used accumulation as a manipulation of impulsiveness. To manipulate strategies, we assigned one individual in each pair to act as a stooge. The stooge was trained to simply "follow lights" and in doing so it could be made to follow an experimentally determined strategy. In this experiment, the stooges either reciprocated using the widely known tit-for-tat strategy (Axelrod and Hamilton, 1981) or unilaterally defected using the "all defect" (All-D). A player using tit-for-tat copies his opponent's move on the previous trial: so if my opponent cooperated on trial 10, I will cooperate on trial 11.

\subsection{Experiment 2 - methods}

\subsubsection{Subjects}

The subjects were eight pairs of adult blue jays (16 birds total). In an effort to maintain similar levels of motivation, we chose birds with similar body weights for each pair. We randomly designated one member of the pair as the "subject" and another as the "stooge." The pairs in this experiment (listed subject first) were: b22 and b18, b24 and b84, b70 and b1, b85 and b140, b122 and b3, b223 and b106, b229 and b130, b239 and b208. In referring to the pairs, we cite only the subject's band number.

\subsubsection{A single trial}

To begin, we describe the sequence of events within a single trial, or play of the game. The computer illuminated the rear lights at the beginning of each trial, signaling that a new trial had started. When both subjects occupied the corresponding rear perches, the rear lights were "washed out" (by switching on an additional white light) to indicate the beginning of the intertrial interval. When the inter-trial interval expired, the apparatus illuminated the subject's front lights, indicating that a choice (or play) had become available. The appropriate front light (as determined by the programmed strategy) for the stooge illuminated at the same time. The trial only proceeded to this choice phase if both individuals occupied their rear perches. Next, both birds hopped forward to one of the front perches; the apparatus extinguished the unchosen light and "washed out" the chosen light (as described above). Once both birds occupied their front perches, the apparatus dispensed food according to experimentally determined game matrices.

\subsubsection{Game matrices}

During tests, the subject experienced the following Prisoner's Dilemma game matrix:

\begin{tabular}{lll}
\hline & \multicolumn{2}{l}{ Stooge } \\
\cline { 2 - 3 } & $\mathrm{C}$ & $\mathrm{D}$ \\
\hline Subject & \\
$\mathrm{C}$ & 4 Pellets & 0 Pellets \\
$\mathrm{D}$ & 6 Pellets & 2 Pellets \\
\hline
\end{tabular}

Students of game theory usually think of the Prisoner's Dilemma game as model of cooperation, so they customarily called one action "cooperate" (denoted by C in the game matrix) and the other option "defect" (meaning "not cooperate," denoted by D).

The game matrix shows how the subject's payoffs depend on the combined actions of the subject and the stooge. For example, if the stooge plays D and the subject plays $\mathrm{C}$ the subject obtains zero pellets (see Mesterton-Gibbons, 1992 for a review of the game matrix and other ideas from game theory). During all trials, the stooge experienced a behaviorally neutral game matrix in which it obtained two pellets for all possible combinations of actions. The reason for this was two-fold. First, it was an effort to maximize our control over the stooge's behavior by offering the stooge the same motivation to act in all circumstances. Second, the stooge would then obtain slightly less food on average than the subject, which again was an effort to ensure that the stooge was at least as motivated to act as the subject. We wanted to avoid situations, for example, in which the stooge was satiated (and so had a high latency to act), while the subject was still hungry.

\subsubsection{Mutualism precedes Prisoner's Dilemma}

Game theoretical equilibria are stability concepts. To test the stability of cooperation, we sought to first create high levels of cooperation. To achieve this, we presented each subject with a mutualism matrix given by:

\begin{tabular}{lll}
\hline & \multicolumn{2}{l}{ Stooge } \\
\cline { 2 - 3 } & C & D \\
\hline Subject & & \\
C & 4 & 2 \\
D & 0 & 0 \\
\hline
\end{tabular}

which quickly led to high levels of the $\mathrm{C}$ response. Subjects experienced this mutualism treatment before each Prisoner's Dilemma test, for a minimum of 3 days, terminating only when the subject showed $80 \% \mathrm{C}$ or higher for two consecutive days.

Following the results of experiment 1, we arranged all trials into clumps of four. The birds waited $345 \mathrm{~s}$ and then played four times in quick succession with $5 \mathrm{~s}$ between plays. In addition, we arranged trials in blocks of 40 (10 
clumps of 4), with 8 forced or no choice trials followed by 32 free choice trials. During the forced choice trials, the apparatus presented only one option to the subjects (either or C or D), while the stooge continued to play its programmed strategy (TFT or All-D).

As explained above, the $\mathrm{C}$ and $\mathrm{D}$ choices were defined by their positions. The $C$ choice was the perch closest to the opponent, while the $\mathrm{D}$ choice was further away. In addition, we changed the color of the stimulus light associated with $\mathrm{C}$ and D for each accumulation/strategy treatment. We used four color pairs - yellow/red, yellow/blue, red/green and green/orange - chosen because they seemed about equally discriminable to a human observer. We randomized the color pair used for each subject in each treatment, assigning one of the two colors to C and the other to D. The stooge's choice light was always green.

\subsubsection{Stooge strategy treatments}

As described above, the stooge implemented a strategy that we determined. We simply programmed the apparatus so that the appropriate choice (C or D) was the only one available to the stooge. For example, in tit-for-tat we programmed the apparatus to show the inside $C$ light if the subject cooperated on the previous trial, and to show the outside D light if the subject defected on the previous trial. The appropriate payoff matrix (mutualism or Prisoner's Dilemma) determined the subject's payoffs, just as if the stooge had chosen freely. When there were long programmed gaps between plays, such as from 1 day to the next, or over the mid-day break, the tit-for-tat player began by cooperating.

\subsubsection{Accumulation treatments}

In accumulated treatments, the apparatus held food in the transparent accumulator (Figure 1B) for a clump of four trials, while in non-accumulated treatments the flapper was opened at the end of each trial, immediately after the pellet dispenser operated.

4.2.6.1. General plan of a treatment. The general plan of a single treatment is straightforward. First, we randomly assigned stimulus colors, stooge strategy and accumulation treatment. Second, the subject experienced the mutualism matrix (with the chosen accumulation and stooge-strategy treatments in force) until the cooperation criterion was satisfied. Third, we changed the subject's payoff matrix to the Prisoner's Dilemma matrix, and this was in force until the subject completed 1000 free choice trials (5-7 days). We repeated this cycle until each subject had completed all four accumulation/ strategy combinations. In a few cases, a subject completed less than 1000 free trials (never less than 700 free trials) because of an error in the program that managed the transitions from one treatment to the next.

\subsection{Experiment 2 - results}

Experiment 2 considered the effect of accumulation and strategy on the stability of cooperation, and we published the main results on this topic previously (Stephens et al., 2002). This paper emphasizes unpublished results on the effect of temporal clumping, but we review the earlier results because they lay the groundwork for our clumping results. The basic result of the experiment was a three-way interaction between opponent strategy, accumulation and time block. This interaction showed that cooperation persisted when the opponent reciprocated (played tit-for-tat) and payoffs accumulated; in contrast, cooperation eroded toward defection in other situations.

As explained in the methods, our procedure clumped trials ("plays" in game-theoretic jargon) together in time because experiment 1 showed that clumping enhanced the effect of accumulation on impulsiveness. Here, we ask whether subjects cooperate more on the first play of clump or on the second and so on. In this re-analysis, therefore, we conducted a large repeated measures analysis of variance, similar to the one used to analyze previously published data, except that we added "clump position" as a fixed factor. Table 1 shows the resulting ANOVA table. The table shows the same pattern of significant and insignificant results as in our earlier published analysis, but also shows two significant effects that involve position within the clump: a significant main effect of clump position and a significant interaction of accumulation and clump position (codes ACCUM and CLMP-POS). Figure 5 shows the accumulation-position interaction. The figure shows that subjects cooperate at roughly the same level in the first play of a clump regardless of whether payoffs are accumulated or not accumulated. However, the level of cooperation drops on the second, third and fourth plays of the clump in both treatments; however, this drop is larger in the unaccumulated treatments. A post hoc analysis using Fisher's least-significance difference tests at the $5 \%$ level of significance suggests that the clump positions 2-4 in the unaccumulated treatments are a distinct subset of the data with markedly lower levels of cooperation. The figure suggests a similar but more subtle decline in accumulated treatments, but post hoc analysis suggests this is not

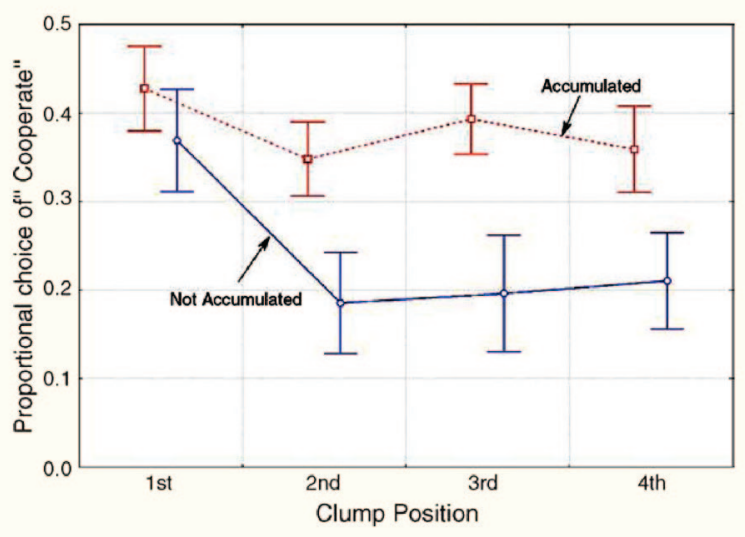

Figure 5. The significant accumulation/clump position interaction $\left(F_{3,21}=7.83, p=0.0011\right)$ observed in experiment 2 . The plot shows the relative frequency of cooperation as a function of a trial's position within a clump of plays (clump position). Subjects cooperated at relatively high rates in the first trial of a clump, but the frequency of cooperation declined markedly on later trials in "Not Accumulated" treatments. In "Accumulated" treatments, the frequency of cooperation did not drop significantly on the 2nd, 3rd and 4th trials of the clump. As in previous figures, this plot summarizes data from the final third of free trails for each treatment. 


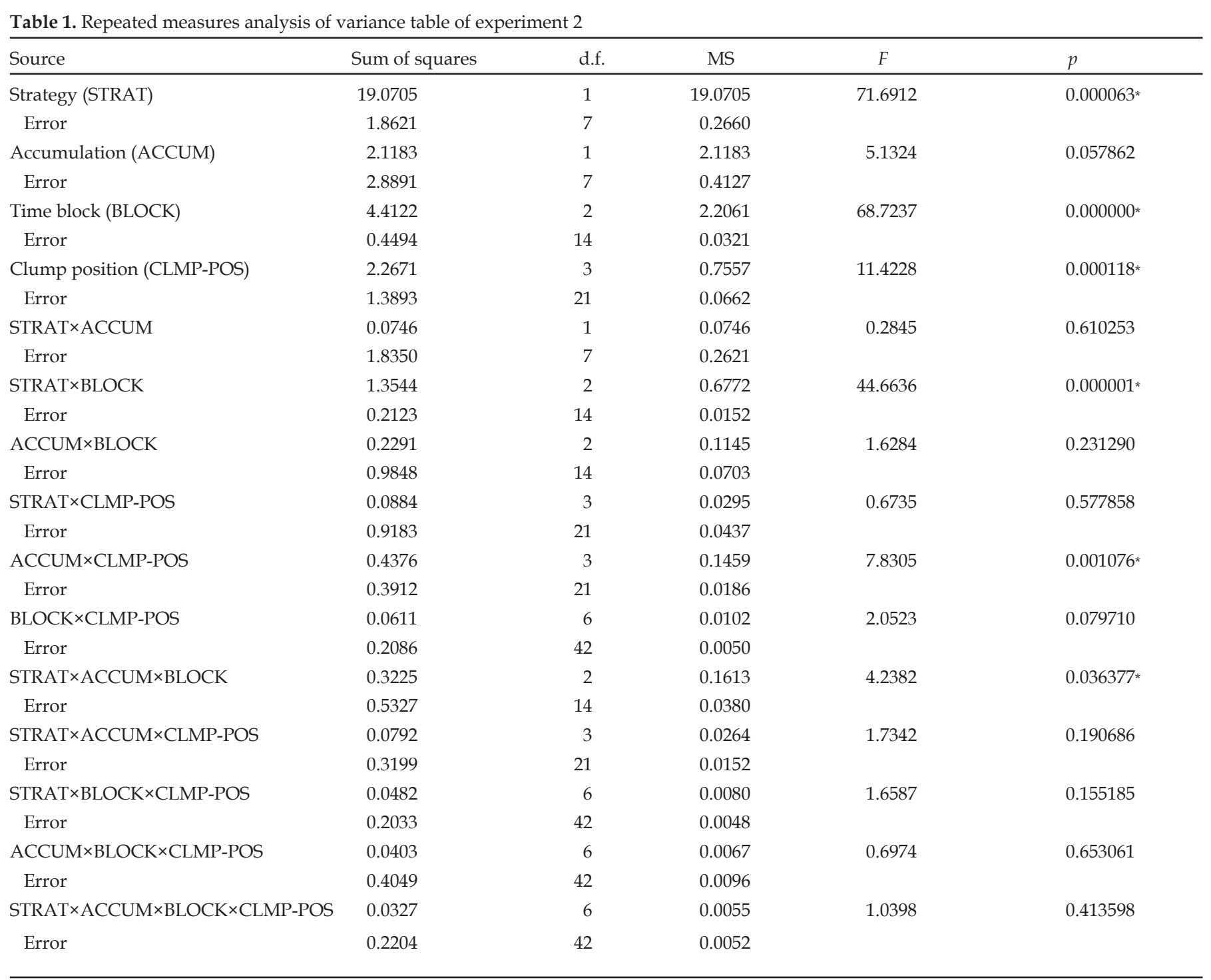

significant. Overall, this new analysis including the effects of clump position, suggests shows that the overall difference in cooperation we reported previously is largely due to differences that occur after the first play of a clump.

We find that subjects cooperate more frequently after the long interval between clumps than after the short interval within clumps, and the drop in cooperation from the first to second play is greatest when we distribute payoffs immediately. We do not have a convincing explanation for this result, but we can offer two plausible interpretations. One possibility is that subjects simply forget their place in the intervals between clumps of plays, and this causes them to fall back to typical behavior, which tends to increase cooperation. Recall that our procedures start each treatment with high levels of cooperation, so that subjects have had more overall experience choosing the " $\mathrm{C}$ " option than the " $\mathrm{D}$ " option - therefore, forgetful subjects might return to the previously reinforced "C" option. The subject needs the first play in a clump to "jog" its memory before it begins to respond appropriately to the opponent on the second play. In this view, the salience of actually receiving food in unac- cumulated treatments jog the subject's memory more effectively than the visible but unavailable food deliveries of the accumulated treatments. The explanation works best for the tit-for-tat treatments, since it is somewhat unclear how a jay can "lose its place" in the all defect treatments where they should always defect as their opponent does.

The second possibly relies on game theory. In the Prisoner's Dilemma, it can never be rational to cooperate when only one play remains, by extension game theorists have long argued that if two players know they will play a fixed number of times they should always defect (see MestertonGibbons, 1992 for a complete discussion). So a second possibility is that subjects view the clumps of four plays like a finitely repeated game, in which they "know" they will play the game exactly four times. To explain a shift to defection within a clump of plays, we assume that a subject's certainty about how many plays remain depends on the position of a trial in the clump. Psychophysics (e.g., Gescheider, 1985) tells us that the more plays remain, the more unsure the subjects should be. Therefore, we would expect fairly confident defection on the last play of a clump. Intuitively the idea is 
that subjects "care about" the four-play future of an interaction at the beginning of a clump of plays, but do not care at all on the last play. This hypothesis does not explain the stepwise transition from the first to second plays as neatly as the forgetting hypothesis, although the forgetting hypothesis, at best, explains only some aspects of our results.

\subsection{Experiment 2-Discussion}

Experiment 2 explored the role of accumulation and strategy in cooperation, and found - as previously reported - that cooperation persisted in treatments where payoffs accumulated and the opponent reciprocated. This paper shows that subjects cooperated most in the first play of a clump with a distinct drop in levels of cooperation after this when payoff did not accumulate. The effects of accumulation on cooperation are due to differences that occur in the second, third and fourth plays of a clump. As in experiment 1, we see that temporal clumping plays an unexpected role in determining the level of cooperation.

\subsubsection{Clumps and cooperation}

Our demonstration that cooperativeness varies with the position of a play within a sequence of clumped plays adds a new level of complexity to models of cooperation. Theoretical approaches to cooperation in social dilemmas typically ignore the temporal organization of plays (see Stephens, 2000; Stephens et al., 1995 for exceptions). For example, modelers have advocated a great many strategies for the Prisoner's Dilemma (tit-for- tat, generous tit-for-tat, Pavlov and so on), but none of these strategies considers the temporal aspects of cooperative games. Moreover, we comment that naturally occurring cooperative situations will be organized in time in many different ways. Some may have a clear bout structure, with bouts of play separated by long interval of non-play (e.g., two male manakins repeatedly dance for a female, but then wait a long time for the next female to arrive). Others may have no specific temporal clumping, but have opportunities to play occurring randomly. The evenly spaced structure of repeated play tacitly assumed by most models of cooperation is perhaps the least likely possibility. Our results suggest that these arrangements may have important effects on the behavioral control of cooperation.

We find that cooperation is a rather fragile phenomenon (Stephens et al., 2002). We had to construct rather specialized circumstances to generate cooperative behavior. To circumvent the problem of impulsiveness, we had to both clump trials and accumulate payoffs. In addition, the jays had to play against strict TFT strategists. Although our clumped/accumulated design does model some natural cooperative situations, it does not reflect all instances of cooperation. These experiments suggest that cognitive constraints such as impulsivity may limit the role of reciprocity as a general mechanism of cooperation (Stevens and Hauser, 2004).

\section{General discussion}

We have considered the combined effects of payoff accumulation and temporal clumping on impulsiveness and coop- eration. Our first experiment shows that subjects were less impulsive when payoffs accumulated, but only when we arranged trials in clumps. We expected accumulation to reduce impulsiveness and reasoned that clumping would enhance this effect. Instead, our data suggest that clumping increases impulsiveness and accumulation eliminates the effect of clumping because jays were most impulsive in our clumped and unaccumulated treatment but about equally impulsive in all other treatments.

\subsection{Calculating long-term rates}

The first sentence of this paper defines impulsivity in terms of rate. We characterize an animal's behavior as impulsive if it chooses a smaller more immediate option even when a larger more delayed option yields a higher long-term rate. Our clumped treatments raise questions about the meaning of "long-term rate" that do not arise in "unclumped" studies of impulsivity. Consider a self-control experiment that offers an animal a choice between a small-immediate option, yielding amount $A 1$ after delay $t 1$, and large-delayed option, yielding amount $A 2$ after delay $t 2$. In addition, the subject waits for inter-trial interval $\tau$ between trials. The conventional way to calculate the highest long-term rate is to compare:

$$
\frac{A_{1}}{\tau+t_{1}} \text { and } \frac{A_{2}}{\tau+t_{2}}
$$

For a situation with evenly-space trials, the quantity $A_{i} /$ $\left(\tau+t_{i}\right)$ is the rate calculated from one choice point to the next (the "choice-to-choice" rate) and the infinite time-horizon rate (the really "long-term" rate). However, if the inter-trial interval varies as it does in our clumped treatments, then the choice-to-choice rate and the long-term rate are not the same. In our clumped treatments, with clumps of four trials, the inter-trial interval for the first trial is $345 \mathrm{~s}$, followed by three very short inter-trial intervals of $5 \mathrm{~s}$. So the choice-tochoice rate for the first trial in a clump is $A_{i} /\left(345+t_{i}\right)$, while the choice-to-choice rate for the second trial in a clump is $A_{i}$ $/\left(5+t_{i}\right)$, but the long-term rate is necessarily the same for all trials. The long-term rate is $A_{i} /\left(90+t_{i}\right)$ because the average inter-trial interval is $90 \mathrm{~s}, 1 / 4 \times 345+3 / 4 \times 5=90$. We designed our study so that the long-term rates, measured in this sense, were the same in our clumped and unclumped treatments.

We comment that one can calculate a different average rate using a weighted average of the choice-to-choice rates: $1 / 4 \times A_{i} /\left(345+t_{i}\right)+3 / 4 \times A_{i} /\left(5+t_{i}\right)$. Most students of feeding behavior now agree, however, that averaging the time and amount components separately and then calculating the quotient of the averages gives a more meaningful estimate of the long-term rate. Specifically this quotient of the averages measures the rate of gain in a typical unit of time, while the weighted average of choice-to-choice rates gives the rate in a typical trial. The literature of rate-based foraging models contains extensive discussions of these issues (Bateson and Kacelnik, 1996; Stephens and Krebs, 1986; Templeton and Lawlor, 1981; Turelli et al., 1982), but there seems to have been little discussion of the problems of calculating rates in a variable world in the literature of impulsivity. 
If one accepts that long-term rates, as explained above, provide the appropriate standard against which to recognize impulsivity, then there can be little doubt that the observed increase in choice of the small-immediate option in clumped treatments represents a change in impulsivity because the alternatives in our clumped and unclumped treatments provide the same long-term rates.

Even if we agree that the long-term rate provides the correct standard of comparison for the definition of impulsivity, a critical reader might ask whether our subjects responded to choice-to-choice rates. This might explain the shift toward the small-immediate option in clumped treatments because the small-immediate option often provides a high choice-tochoice rate in clumped treatments. However, if choice-tochoice rates governed our jay's choice behavior we should have observed different behavior in the last trial. Specifically, we would expect preference for large-delayed in the last trial of clump and preference for small-immediate on the first, second and third trials. As reported above, experiment 1 found no effect of clump position on choice behavior. So, if sensitivity to choice-to-choice rates influenced our jays, they made a mistake by over-emphasizing the three trials that occur in quick succession and underemphasizing the long-wait to the beginning of the next clump.

\subsection{Limitations and further questions}

As with any novel result, one must ask how general these clumping effects might be. To answer this question, we need further studies on the effects of clumping on impulsiveness. Our studies differ from most studies of impulsivity because we ran $8 \mathrm{~h}$ sessions and subjects obtained nearly all of their food from the apparatus. In this procedure, a session fills an $8 \mathrm{~h}$ day and trials occur on a longer time scale than in more common open economy procedures. A clump of trials may be a more salient feature of our long sessions than it would be in typical open-economy procedures. Assuming for the moment that further studies validate our clumping result, we want to know why it occurs. The hypothesis that variability in the inter-trial interval (clumping) increases the background value suggests several further studies. An obvious question is whether random variation in the intertrial interval has the same effect as the deterministic variation we have studied.

Similar concerns arise in connection with the clump-position effect on cooperation that we report. It is striking that we do not find similar clump-position effects in experiment 1 , where we focused on impulsiveness and not cooperation. Of course, a key difference between the studies is that the stooge's experimentally determined strategy creates sequential relationships between successive plays. Manipulations of the stooge's strategy and interval between clumps could be revealing here.

\subsection{Summary}

We present two experiments that investigate the role of payoff accumulation and temporal clumping of trials in impulsiveness and cooperation. Our results show that clumping makes animals more impulsive, but that payoff accumula- tion can eliminate this effect. Although animal impulsiveness is a widely studied phenomenon, we believe that this study is the first to report this clumping effect. In addition, this clumping effect is one of only two results showing that aspects of the choice background can influence impulsiveness. We also show that cooperativeness varies with the position of trial within a clump of trials. Taken together, these results suggest that temporal clumping and related issues of the temporal organization of the choices may provide important new insights into impulsiveness and cooperation.

Acknowledgments - NIMH (R01-MH64151-01) supported this research. We thank our colleagues Matt Scott, Julian MacDonald, Aimee Dunlap-Lehtila, Claire Leung, Jolene Ruf and many other members of blue jay lab for their assistance and support. We thank Jesse Dallery for thoughtful and constructive comments.

\section{References}

Ainslie, G., Monterosso, J. R., 2003. Building blocks of self-control: increased tolerance for delay with bundled rewards. J. Exp. Anal. Behav. 79, 37-48.

Axelrod, R., Hamilton, W. D., 1981. The evolution of cooperation. Science 211, 1390-1396.

Bateson, M., Kacelnik, A., 1996. Rate currencies and the foraging starling: the fallacy of the averages revisited. Behav. Ecol. 7, 341-352.

Brunner, D., Gibbon, J., 1995. Value of food aggregates: parallel versus serial discounting. Anim. Behav. 50, 1627-1634.

Clements, K. C., Stephens, D. W., 1995. Testing models of non-kin cooperation: mutualism and the prisoner's dilemma. Anim. Behav. 50, 527-535.

Gallistel, C. R., Gibbon, J., 2000. Time, rate, and conditioning. Psychol. Rev. 107 (2), 289-344.

Gescheider, G. A., 1985. Psychophysics: Method, Theory and Application, second ed. L. Erlbaum Associates, Hillsdale, NJ.

Green, L., 2000. Self control, impulsivity and risk taking: psychological and economic accounts of discounting delayed and probabilistic rewards. Int. J. Psychol. 35 (3-4), 114.

Green, L., Fisher Jr., E. B., Perlow, S., Sherman, L., 1981. Preference reversal and self-control: choice as a function of reward amount and delay. Behav. Anal. Lett. 1, 244-256.

Harris, A. C., Madden, G. J., 2002. Delay discounting and performance on the prisoner's dilemma game. Psychol. Rec. 52 (4), 429-440.

Jackson, K., Hackenberg, T. D., 1996. Token reinforcement, choice and self-control in pigeons. J. Exp. Anal. Behav. 66, 29-49.

Mazur, J. E., 1986. Choice between single and multiple delayed reinforcers. J. Exp. Anal. Behav. 46, 67-77.

Mazur, J. E., 1987. An adjusting procedure for studying delayed reinforcement. In: Commons, M. L., Mazur, J. E., Nevin, J. A., Rachlin, H. (eds.), Quantitative Analyses of Behavior: The Effect of Delay and of Intervening Events on Reinforcement Value. Lawrence Erlbaum, Hillsdale, NJ, pp. 55-73.

Mazur, J. E., 1989. Theories of probabilistic reinforcement. J. Exp. Anal. Behav. 51, 87-99.

Mazur, J. E., 1994. Effects of intertrial reinforcers on self-control choice. J. Exp. Anal. Behav. 61, 83-96. 
Mazur, J. E., Logue, A. W., 1978. Choice in a "self-control" paradigm: effects of a fading procedure. J. Exp. Anal. Behav. 30, 11-17.

Mazur, J. E., Snyderman, M., Coe, D., 1985. Influences of delay and rate of reinforcement on discrete-trial choice. J. Exp. Psychol. Anim. Behav. Process. 11, 565-575.

Mesterton-Gibbons, M. (1992). An Introduction to Game-theoretic Modelling. Redwood City, CA.

Mischel, W., Shoda, Y., Rodriguez, M. L., 1989. Delay of gratification in children. Science 244, 933-938.

Mitchell, S. H., Rosenthal, A. J., 2003. Effects of multiple delayed rewards on delay discounting in an adjusting procedure. Behav. process. $64,273-286$.

Platt, J., 1973. Social traps. Am. Psychol. 28, 641-651.

Rachlin, H., 2000. The Science of Self-control. Harvard University Press, Cambridge, MA.

Rachlin, H., 2002. Altruism and selfishness. Behav. Brain Sci. 25, 239- 296.

Rachlin, H., Brown, J., Cross, D., 2000. Discounting in judgments of delay and probability. J. Behav. Decis. Making 13 (2), 145-159.

Rachlin, H., Green, L., 1972. Commitment, choice and self-control. J. Exp. Anal. Behav. 17, 15-22.

Rachlin, H., Raineri, A., Cross, D., 1991. Subjective probability and delay. J. Exp. Anal. Behav. 55, 233-244.

Richards, J. B., Mitchell, S. H., de Wit, H., Seiden, L. S., 1997. Determination of discount functions in rats with an adjustingamount procedure. J. Exp. Anal. Behav. 67, 353-366.

Sally, D., 1995. Conversation and cooperation in social dilemmas: a meta-analysis of experiments from 1958 to 1992. Ration. Soc. 7, 58-92.
Stephens, D. W., 2000. Cumulative benefit games: achieving cooperation when players discount the future. J. Theor. Biol. 205 (1), 1-16.

Stephens, D. W., Anderson, D., 2001. The adaptive value of preference for immediacy: when shortsighted rules have farsighted consequences. Behav. Ecol. 12 (3), 330-339.

Stephens, D. W., Krebs, J. R., 1986. Foraging Theory. Princeton University Press, Princeton, NJ.

Stephens, D. W., McLinn, C. M., 2003. Choice and context: testing a simple short-term choice rule. Anim. Behav. 66, 59-70.

Stephens, D. W., McLinn, C. M., Stevens, J. R., 2002. Discounting and reciprocity in an iterated prisoner's dilemma. Science 298, 2216-2218.

Stephens, D. W., Nishimura, K., Toyer, K. B., 1995. Error and discounting in the iterated prisoner's dilemma. J. Theor. Biol. 176, 457-469.

Stevens, J. R., Hauser, M., 2004. Why be nice? Psychological constraints on the evolution of cooperation. Trends Cogn. Sci. 8, 60-65.

Templeton, A. R., Lawlor, L. R., 1981. The fallacy of averages in ecological optimization theory. Am. Nat. 117, 390-393.

Turelli, M., Gillespie, J. H., Schoener, T. W., 1982. The fallacy of the fallacy of the averages in ecological optimization theory. Am. Nat. 119, 879-884.

Wilson, M., Daly, M., 2004. Do pretty women inspire men to discount the future? Proc. R. Soc. Lond. B 271 (Suppl.), 177-179 